\title{
Time-dependent reliability of (weighted) $k$-out-of- $n$ systems with identical component deterioration
}

\author{
Cao Wang (D)
}

\begin{abstract}
The performance of civil infrastructure systems is vital in supporting a community's functionalities. Reliability assessment of these systems is a powerful approach to evaluate whether the system performance is desirably safe under the impacts of resistance degradation and non-stationary loads. A k-out-of- $n$ system is a widely-used logic model for a system with $n$ components, which survives (works) if at least $k$ components work. Its special cases include a series or a parallel system. Furthermore, a weighted k-out-of- $n$ system has components with positive integer weights and the system survives if the total weight of working components reaches the predefined threshold $k$. This paper proposes a method for estimating the time-dependent reliability of both ordinary and weighted k-out-of- $n$ systems, taking into account the effects of resistance deterioration, resistance correlation and load non-stationarity, for which a mathematical solution is derived. The applicability of the proposed method is illustrated through reliability evaluation of a representative $k$-out-of- $n$ system.
\end{abstract}

Keywords: System reliability, Time-dependent reliability, Weighted k-out-of-n system, Degradation, Non-stationary loads

\section{Introduction}

Civil infrastructure systems are expected to function with an acceptable level of serviceability and safety during their service lives. The aggressive environmental or operating conditions in service, however, could threaten the system performance significantly. Taking into account the uncertainties associated with these safety-threatening factors, which are often difficult or even impossible to predict exactly, structural reliability assessment is a powerful tool to evaluate a system's capability of fulfilling the safety requirements during a reference period of interest [1-4].

An important ingredient in reliability assessment is to model the degradation of structural resistance (e.g., strength, stiffness, and others) at both the component and system levels [5-8]. On the other hand, the external load process could be non-stationary on the temporal scale in

Correspondence: wangc@uow.edu.au

School of Civil, Mining and Environmental Engineering, University of Wollongong, Wollongong, NSW, Australia terms of occurrence frequency and/or magnitude [9-11]. For example, for structures in cyclone-prone areas, the future cyclone winds could be affected by the impact of climate change $[9,10]$. As a result, it is important to incorporate the factors of resistance deterioration and load non-stationarity in structural reliability assessment. Mori and Ellingwood [12] developed a method for estimating structural time-dependent reliability, where the load process was modeled by a homogeneous Poisson process. This work was later improved by Li et al [13] so that the non-stationarity in the load process can also be considered in structural reliability assessment.

Most of infrastructure systems consist of multiple structures or components [14]. One of the key features of a system is the interaction between different components [15-17]. Furthermore, correlation may also arise between the performances of different components due to common design provisions and construction practices $[18,19]$. Wang et al [20] developed an approach for estimating the 
time-dependent reliability of an aging series system considering resistance correlation of different components. Wang and Zhang [21] investigated the seismic resilience of a power grid system, where the impact of correlation and deterioration of component resistances was considered.

The concept of a $k$-out-of- $n$ system has been extensively employed in engineering practice, which refers to such a system with totally $n$ components that the system survives (works) if at least $k$ components normally work. With this definition, the system is written as a $k$-out-of- $n: G$ system. Intuitively, the system reduces to a series or parallel system when $k=n$ or $k=1$ respectively. Note that there exists an alternative definition for $k$-out-of- $n$ system, with which the system fails if at least $k$ components fail. Both definitions serve as a complement for each other and should be used with careful instruction. In this paper, the $k$-out-of- $n$ : $G$ system will be discussed, and will be simply referred to as a $k$-out-of- $n$ system unless otherwise stated.

A generalized case of a $k$-out-of- $n$ system is known as a weighted $k$-out-of- $n$ system [22], where each component has a positive integer weight. Correspondingly, the system works when the total weight of working components reaches the threshold $k$. For example, consider two substations from an electricity distribution network, which are connected directly by different transmission lines (components) [23]. The voltage of each transmission line could differ, resulting in different weights accordingly. When a certain level of voltage is needed for electricity transmission between the two substations, giving the required threshold $k$, the system (consisting of these transmission lines) can be modeled by a weighted $k$-out-of- $n$ system. In the presence of natural hazards such as earthquakes, the resistance of each component is reflected by the seismic fragility curve, which could degrade due to the impact of environmental conditions [21]. One can also refer to [24] for other examples of a weighted $k$-out-of- $n$ system. When the weight for each component equals unity, the weighted $k$-out-of- $n$ system reduces to an ordinary one.

The reliability assessment of a $k$-out-of- $n$ system has been widely discussed in previous studies [25-27]. Wang et al [28] proposed a method for time-dependent reliability of a $k$-out-of- $n$ system with common cause failure through system-level load-strength interference analysis. Zhang et al [29] developed a reliability model for a loadsharing $k$-out-of- $n$ system subjected to discrete loads, where the load-sharing effect was reflected by modeling the load distribution variation and strength damage after component failures directly. However, these studies did not consider the impacts of component resistance (in terms of correlation and deterioration) and load nonstationarity on the time-dependent reliability of $k$-out-of$n$ systems.

In an attempt to compute the reliability of a weighted $k$-out-of- $n$ system, recursive formulas were developed in
[30, 31]. Eryilmaz and Tutuncu [32] investigated the reliability of a weighted $k$-out-of- $n$ system by modeling the component interdependency as a Markov type. Eryilmaz [33] studied the reliability of a $k$-out-of- $n$ system with random weights and proposed a recursive formula to compute the system state probabilities. Faghih-Roohi et al [34] presented a dynamic model for availability assessment of multi-state weighted $k$-out-of- $n$ system and optimized the component availability and capacity through a genetical algorithm. Coit et al [35] proposed a reliability model for dynamic $k$-out-of- $n$ system considering component partnership, where the system reliability at time $t$ is measured by the instantaneous system performance. Franko et al [36] studied the impact of cold standby component on the reliability of weighted $k$-out-of- $n$ systems with two types of components. Zhang [37] performed reliability analysis of $k$-out-of- $n$ systems with heterogeneous components and random weights. Hamdan et al [38] developed an optimal preventive maintenance model for weighted $k$-out-of- $n$ systems on the basis of cost analysis. Yet the time-dependent reliability assessment of a weighted $k$-out-of- $n$ system has to be addressed by considering the time-variation of both component resistances and external loads.

This paper presents a method for the time-dependent reliability of an aging (weighted) $k$-out-of- $n$ system, taking into account the impacts of component resistance deterioration, resistance correlation and non-stationary load effect. It is shown that the proposed reliability method is a generalized form of that for a single component, series system or parallel system. The implementation of the proposed method is also discussed. The applicability of the developed reliability method is demonstrated through an illustrative example.

\section{Time-dependent reliability assessment Reliability of a single component}

Consider the reliability of a component over time interval $[0, T]$. Significant load events (e.g., earthquake events) occur randomly in time with random intensities. Using a Poisson process to model the occurrence of the load events, let $\lambda(t)$ be the time-variant occurrence rate $(t$ denotes time), and $F_{S}(s, t)$ the cumulative distribution function (CDF) of the load effect at time $t$ conditional on occurrence. Mathematically, if the load effect at time $t$ is $S(t)$, then $\operatorname{Pr}(S(t) \leq s)=F_{S}(s, t)$, where $\operatorname{Pr}()$ denotes the probability of the event in the brackets.

The resistance at time $t, R(t)$, is modeled as follows,

$$
R(t)=R_{0} \cdot g(t)
$$

where $g(t)$ is the deterioration function, and $R_{0}$ is the initial resistance. The deterioration function may take different shapes (e.g., linear, square-root and parabolic), 
depending on the dominant deterioration mechanism $[2,39]$.

With this, the time-dependent reliability within $[0, T]$, $L(T)$, is estimated by [13]

$$
\begin{gathered}
L(T)=\int_{0}^{\infty} \exp \left\{-\int_{0}^{T} \lambda(t)\left[1-F_{S}(r \cdot g(t), t)\right] \mathrm{d} t\right\} \\
\cdot f_{R}(r) \mathrm{d} r
\end{gathered}
$$

where $f_{R}(r)$ is the probability density function (PDF) of the initial resistance.

\section{Reliability of a series or parallel system}

For a series system with $n$ components (structures), let $R_{j}$ be the initial resistance of component $j(j=1,2, \ldots n)$, and $g_{j}(t)$ the deterioration function of component $j=$ $1,2, \ldots n$, which is assumed to be independent of the load process. Suppose that the load process is modeled by a Poisson process with a time-variant occurrence rate of $\lambda(t)$ and a time-variant CDF of load effect $F_{S}(s, t)$. An occurring load event with magnitude $s$ induces a structural action $c_{j} \cdot s$ in component $j$ (e.g., moment, shear, etc) for $j=1,2, \ldots n$. With this, the time-dependent series system reliability, $L_{s s}(T)$, is given by [20]

$L_{s s}(T)=\int \ldots \int \exp \left[-\int_{0}^{T} \lambda(t) \cdot\left\{1-F_{S}\left(\min _{j=1}^{n} \frac{r_{j} g_{j}(t)}{c_{j}}, t\right)\right\} \mathrm{d} t\right] f_{\mathbf{R}}(\mathbf{r}) \mathrm{d} \mathbf{r}$

where $f_{\mathbf{R}}(\mathbf{r})$ is the joint PDF of the initial resistances $\mathbf{R}=$ $\left\{R_{1}, R_{2}, \ldots R_{n}\right\}$, and $\mathbf{r}=\left\{r_{1}, r_{2}, \ldots r_{n}\right\}$.

For a parallel system consisting of $n$ components with the same configuration as that of the series system as discussed earlier, if each $c_{j}$ is independent of the number of working components, and the deterioration function of each component is identical, denoted by $g(t)$, the timedependent parallel system reliability, $L_{p s}(T)$, is given by [40]

$L_{p s}(T)=\int \ldots \int \exp \left[-\int_{0}^{T} \lambda(t) \cdot\left\{1-F_{S}\left(\max _{j=1}^{n} \frac{r_{j} g(t)}{c_{j}}, t\right)\right\} \mathrm{d} t\right] f_{\mathbf{R}}(\mathbf{r}) \mathrm{d} \mathbf{r}$

In Eqs. (3) and (4), if the component resistances are statistically independent, then $f_{\mathbf{R}}(\mathbf{r})=\prod_{i=1}^{n} f_{R_{i}}\left(r_{i}\right)$, where $f_{R_{i}}\left(r_{i}\right)$ is the PDF of $R_{i}$. However, taking into account the correlation between different component resistances, one would need to reasonably model the probabilistic behaviour of $f_{\mathbf{R}}(\mathbf{r})$. With this regard, the copula function is a promising tool to describe correlated random variables $[40,41]$.

\section{Reliability of a $k$-out-of- $n$ system}

For a $k$-out-of- $n$ system with $n$ components (structures), the system survives if at least $k$ components work, as mentioned before. Its time-dependent reliability will be discussed in this section. Assume that the resistance deterioration function for each component is identical, denoted by $g(t)$, and is independent of the load process. It is also assumed that the value of $c_{j}(j=1,2, \ldots n)$ is independent of the number of working components.

We introduce $\mathcal{M}(\mathbf{a}, k)$, which is a function of vector a (with $n$ elements) and integer $k \leq n$. The function $\mathcal{M}$ returns the $k$ th largest element of a, which will be used in the following derivation. It is straightforward to observe that $\mathcal{M}(\mathbf{a}, 1)=\max (\mathbf{a})$ and $\mathcal{M}(\mathbf{a}, n)=\min (\mathbf{a})$.

The hazard function, denoted by $h(t)$ at time $t$, represents the probability of structural failure at time $t$ provided structural survival up to time $t$. It can be related to the time-dependent reliability $L(t)$ according to $[3,40]$

$$
h(t)=-\frac{\mathrm{d} \ln L(t)}{\mathrm{d} t}
$$

or equivalently,

$$
L(t)=\exp \left(-\int_{0}^{t} h(\tau) \mathrm{d} \tau\right)
$$

Consider the hazard function $h_{k n}(t)$ for the $k$-out-of- $n$ system at time $t$, conditional on $\mathbf{R}=\left\{R_{1}, R_{2}, \ldots R_{n}\right\}=\mathbf{r}=$ $\left\{r_{1}, r_{2}, \ldots r_{n}\right\}$. Let $\mathbf{a}(t)=\left[\frac{r_{1} g(t)}{c_{1}}, \frac{r_{2} g(t)}{c_{2}}, \ldots \frac{r_{n} g(t)}{c_{n}}\right]$. By the definition of hazard function, for $\Delta t \rightarrow 0, h_{k n}(t) \Delta t$ equals the probability that the system fails within time interval $(t, t+\Delta t]$ provided the system's survival within $[0, t]$. The system failure is caused by the occurrence of a significant load event with probability $\lambda(t) \Delta t$, with which

$$
\begin{aligned}
h_{k n}(t) & =\lambda(t)\{1-\operatorname{Pr}[S(t) \leq \mathcal{M}(\mathbf{a}(t), k)]\} \\
& =\lambda(t)\left\{1-F_{S}[\mathcal{M}(\mathbf{a}(t), k), t]\right\}
\end{aligned}
$$

where $S(t)$ is the load effect at time $t$ conditional on load occurrence as before. It is noticed that in Eq. (7), $h_{k n}(t)$ represents the instantaneous failure probability of the system. The condition of system survival before time $t$ refers to the case that at least $k$ components work at any time $\tau \in[0, t]$ (not necessarily all the $n$ components working at time $\tau$ ).

Remark 1 Letting $\mathcal{N}_{f}(\tau)$ denote the number of working components at time $\tau$, the probability of structural survival up to time $t, L(t)$, is determined by

$$
L(t)=\operatorname{Pr}\left(\mathcal{N}_{f}(\tau) \geq k, \forall \tau \in[0, t]\right)
$$

If there are $n_{t}$ load events occurring within $[0, t]$ at times $t_{1}, t_{2}, \ldots t_{n_{t}}$ respectively, it follows that

$$
L(t)=\operatorname{Pr}\left(\bigcap_{i=1}^{n_{t}} S\left(t_{i}\right) \leq \mathcal{M}\left(\mathbf{a}\left(t_{i}\right), k\right)\right)
$$


Eq. 9 is explained by the fact that, with an identical deterioration function for each component, the condition of $\bigcap_{i=1}^{n_{t}} S\left(t_{i}\right) \leq \mathcal{M}\left(\mathbf{a}\left(t_{i}\right), k\right)$ guarantees that the $k$ components with the greatest resistances at the initial time survive at any time $\tau_{1} \in[0, t]$. Furthermore, at time $\tau_{2} \in$ $(t, t+\mathrm{d} t]$, where $\mathrm{d} t \rightarrow 0$, if one load event occurs (with probability $\lambda(t) \mathrm{d} t)$ and the magnitude satisfies $S\left(\tau_{2}\right)>$ $\mathcal{M}\left(\mathbf{a}\left(\tau_{2}\right), k\right)$, then the system fails at time $\tau_{2}$.

According to Eq. (7), the time-dependent reliability for a reference period of $[0, T]$, conditional on $\mathbf{R}=\mathbf{r}$, is estimated by

$$
L_{k n}(T)=\exp \left[-\int_{0}^{T} \lambda(t) \cdot\left\{1-F_{S}[\mathcal{M}(\mathbf{a}(t), k), t]\right\} \mathrm{d} t\right]
$$

which is further rewritten as follows taking into account the uncertainties associated with the component initial resistances,

$$
L_{k n}(T)=\int \ldots \int \exp \left[-\int_{0}^{T} \lambda(t) \cdot\left\{1-F_{S}[\mathcal{M}(\mathbf{a}(t), k), t]\right\} \mathrm{d} t\right] f_{\mathbf{R}}(\mathbf{r}) \mathrm{d} \mathbf{r}
$$

where $f_{\mathbf{R}}(\mathbf{r})$ is the joint PDF of $\mathbf{R}=\left\{R_{1}, R_{2}, \ldots R_{n}\right\}$, and $\mathbf{r}=\left\{r_{1}, r_{2}, \ldots r_{n}\right\}$ as before. Furthermore, Eq. (11) can be simplified as follows,

$L_{k n}(T)=\int \exp \left[-\int_{0}^{T} \lambda(t) \cdot\left\{1-F_{S}[r \cdot g(t), t]\right\} \mathrm{d} t\right] f_{\mathcal{M}(\mathbf{a}(0), k)}(r) \mathrm{d} r$

where $f_{\mathcal{M}(\mathbf{a}(0), k)}(r)$ is the PDF of $\mathcal{M}(\mathbf{a}(0), k)$. Eq. 12 implies that the $(n+1)$-fold integral in Eq. (11) can be converted into a two-fold integral if $f_{\mathcal{M}(\mathbf{a}(0), k)}(r)$ is known. In Eq. (12), if treating $\mathcal{M}(\mathbf{a}(0), k)$ as a generalized initial resistance, then Eq. (12) is consistent with Eq. (2) by considering an equivalent component having a resistance of $\mathcal{M}(\mathbf{a}(0), k)$ for the system.

Eq. 12 (or (11)) is the proposed method for timedependent reliability of an aging $k$-out-of- $n$ system, where the non-stationarity of loads can be reflected by the timevariation of $\lambda(t)$ and $F_{S}(\cdot, t)$.

Remark 2 Note that Eq. (11) is consistent with Eqs. (3) and (4), since a series system is equivalent to an n-out-of-n system and a parallel system is simply a 1-out-of-n system. In fact, in Eq. (11), letting $k=n$ gives $\mathcal{M}(\mathbf{a}(t), k)=$ $\min _{j=1}^{n} \frac{r_{j} g(t)}{c_{j}}$, and similarly, $\mathcal{M}(\mathbf{a}(t), 1)=\max _{j=1}^{n} \frac{r_{j} g(t)}{c_{j}}$.

Remark 3 For an n-dimensional vector a and an integer $k \leq n$, if $\overline{\mathcal{M}}(\mathbf{a}, k)$ returns the kth smallest element of $\mathbf{a}$, then $\mathcal{M}(\mathbf{a}, k)=\overline{\mathcal{M}}(\mathbf{a}, n+1-k)$ and $\overline{\mathcal{M}}(\mathbf{a}, k)=$ $\mathcal{M}(\mathbf{a}, n+1-k)$. The implementation of $\mathcal{M}$ and $\overline{\mathcal{M}}$ can be realized through some commercial software such as Matlab (https://www.mathworks.com). For example, the built-in function mink $(a, k)$ in Matlab returns a vector containing the $k$ smallest elements of a. Illustratively, if $\mathbf{a}=\left[\begin{array}{lllll}1 & 2 & 3 & 4 & 5\end{array}\right]$, then $\mathcal{M}(\mathbf{a}, 2)=\overline{\mathcal{M}}(\mathbf{a}, 4)=$ $\max (\operatorname{mink}(a, 4))=4$.

Remark 4 Note that Eq. (12) has been based on a k-outof- $n$ : G system. If considering a k-out-of- $n: F$ system (that is, the system fails if at least $k$ components fail), then the hazard function for the system is

$$
\begin{aligned}
h_{k n}(t) & =\lambda(t)\{\operatorname{Pr}[S(t)>\overline{\mathcal{M}}(\mathbf{a}(t), k)]\} \\
& =\lambda(t)\left\{1-F_{S}[\overline{\mathcal{M}}(\mathbf{a}(t), k), t]\right\} \\
& =\lambda(t)\left\{1-F_{S}[\mathcal{M}(\mathbf{a}(t), n+1-k), t]\right\}
\end{aligned}
$$

which is consistent with that in Eq. (7).

Remark 5 It is noticed that in Eq. (12), the deterioration process of each component has been assumed to be deterministic. This is applicable for many engineering cases where the variation of the deterioration process is small [42]. However, for a deterioration process with large variation, Eq. (12) needs to be modified slightly to incorporate the effect of uncertainty associated with component deterioration. With this regard, it was shown in [13] that a fully-correlated process is reasonable to describe the component resistance deterioration. That being the case, letting $f_{G(T)}(g)$ be the PDF of $g(T)$, the two-fold integral in Eq. (12) would become three-fold by replacing the item $f_{\mathcal{M}(\mathbf{a}(0), k)}(r) \mathrm{d} r$ with $f_{\mathcal{M}(\mathbf{a}(0), k)}(r) f_{G(T)}(g) \mathrm{d} g \mathrm{~d} r$, if taking into account the uncertainty associated with the component deterioration process.

\section{Reliability of a weighted $k$-out-of- $n$ system}

In this section, the time-dependent reliability of a weighted $k$-out-of- $n$ system will be discussed, which is by nature a generalized form of Eq. (12). Recall that the system works if the sum of weights associated with the working components is no less than the threshold $k$.

As before, let $\mathbf{a}(t)=\left[\frac{r_{1} g(t)}{c_{1}}, \frac{r_{2} g(t)}{c_{2}}, \ldots \frac{r_{n} g(t)}{c_{n}}\right]$, and $\mathbf{a}_{s}(t)$ the sorted $\mathbf{a}(t)$ in a descending order. Let $\mathbf{b}$ be an $n$ dimensional vector representing the weights of the elements in $\mathbf{a}_{s}(0)$. We can determine such a positive integer $k_{s}$ that

$$
k_{s}=\min \left\{j: \sum_{i=1}^{j} b_{i} \geq k\right\}
$$

Clearly, $k_{s}=k$ if $b_{i}=1$ for $\forall i=1,2, \ldots n$.

Note that $\mathcal{M}\left(\mathbf{a}(t), k_{s}\right)=\mathcal{M}\left(\mathbf{a}_{s}(t), k_{s}\right)$. With this, the hazard function $h_{w k n}(t)$ at time $t$, conditional on $\mathbf{R}=\mathbf{r}$, is determined by 


$$
\begin{aligned}
h_{w k n}(t) & =\lambda(t)\left\{1-\operatorname{Pr}\left[S(t) \leq \mathcal{M}\left(\mathbf{a}(t), k_{s}\right)\right]\right\} \\
& =\lambda(t)\left\{1-F_{S}\left[\mathcal{M}\left(\mathbf{a}(t), k_{s}\right), t\right]\right\}
\end{aligned}
$$

Similar to Eq. (10), the time-dependent reliability for a service period of $[0, T]$, conditioned on $\mathbf{R}=\mathbf{r}$, is estimated by

$$
L_{w k n}(T)=\exp \left[-\int_{0}^{T} \lambda(t)\left\{1-F_{S}\left[\mathcal{M}\left(\mathbf{a}(t), k_{s}\right), t\right]\right\} \mathrm{d} t\right]
$$

which, using the law of total probability, is rewritten as follows considering the uncertainty of $\mathbf{R}$,

$$
L_{w k n}(T)=\int \ldots \int \exp \left[-\int_{0}^{T} \lambda(t) \cdot\left\{1-F_{S}\left[\mathcal{M}\left(\mathbf{a}(t), k_{s}\right), t\right]\right\} \mathrm{d} t\right] f_{\mathbf{R}}(\mathbf{r}) \mathrm{d} \mathbf{r}
$$

By referring to Eq. (12), Eq. (17) can be further reduced to

$$
L_{w k n}(T)=\int \exp \left[-\int_{0}^{T} \lambda(t) \cdot\left\{1-F_{S}[r \cdot g(t), t]\right\} \mathrm{d} t\right] f_{\mathcal{M}\left(\mathbf{a}(0), k_{s}\right)}(r) \mathrm{d} r
$$

where $f_{\mathcal{M}\left(\mathbf{a}(0), k_{s}\right)}(r)$ is the PDF of $\mathcal{M}\left(\mathbf{a}(0), k_{s}\right)$.

Eq. 18 (or (17)) presents the proposed time-dependent reliability method for a weighted $k$-out-of- $n$ system in the presence of resistance deterioration and correlation. It has a similar form to Eq. (12) except the item $k_{s}$ involved, due to the impact of the weights for each component.

\section{Implementation of time-dependent reliability assessment}

For the (weighted) $k$-out-of- $n$ system reliability in Eqs. (11) and (17), if the expressions of $f_{\mathcal{M}(\mathbf{a}(0), k)}(r)$ and $f_{\mathcal{M}\left(\mathbf{a}(0), k_{s}\right)}(r)$ are unaccessible, one would need to solve the multi-fold integrals numerically. This is usually time-consuming to conduct, and one could alternatively assess the reliability via Monte Carlo simulation, which is especially powerful in dealing with multi-dimensional problems with mathematical simplicity and robustness $[20,40,43,44]$. Technically, one can first generate a sample for $\mathbf{R}$, denoted by $\mathbf{r}$, and then compute the cores of Eqs. (11) and (17) numerically with $\mathbf{R}=\mathbf{r}$, with which the multi-fold integration can be evaluated via simulation according to

$$
L_{*}(T)=\mathrm{E}\left\{L_{*}(T \mid \mathbf{R})\right\}
$$

where $*=k n$ or $w k n$, and $\mathrm{E}()$ denotes the mean value of the random variable in the brackets.

\section{Illustrative example}

In this section, an illustrative $k$-out-of- $n$ system will be used to show the applicability of the proposed method in Eqs. (12) and (18).
Table 1 Statistics of resistance and load effects of a single component

\begin{tabular}{llll}
\hline Item & Mean $\mathbf{( k N \cdot \mathbf { m } )}$ & $\begin{array}{l}\text { Coefficient } \\
\text { of variation }\end{array}$ & Distribution \\
\hline Initial resistance & 3600 & 0.2 & Lognormal \\
Dead load & 700 & $/$ & Deterministic \\
Live load 1 (LL1) & 500 & 0.35 & Extreme Type I \\
Live load 2 (LL2) & $\begin{array}{l}500(1+0.003 t), t \\
\text { in years }\end{array}$ & 0.35 & Extreme Type I \\
\hline
\end{tabular}

\section{Structural configuration}

Consider a $k$-out-of- 5 system with totally five components, which have identical physical configuration and load conditions. Table 1 presents a summary of the probabilistic models of resistance and loads (dead load and live load). Two live load models, namely LL1 and LL2, are considered, which are associated with an occurrence rate of 1.0 /year. The first is representative of a stationary load process, and the second is a non-stationary load process with an increasing mean load magnitude. Suppose that each load event will induce identical load effect to each component, with which $c_{j}=1$ for $j=1,2, \ldots 5$. The deterioration of resistance for each component is identical and deterministic, taking a form of $g(t)=1-\eta t^{\alpha}$, where $\eta$ and $\alpha$ are two parameters reflecting the rate and shape of the deterioration process. The deterioration function evaluated at the end of 50 years equals 0.8 . The initial resistances of the components are identically distributed and equally correlated pairwise with a correlation coefficient of $\rho=0.5$, unless noted otherwise. The Gaussian copula is used to describe the joint behaviour of different component resistances at the initial time.

\section{System reliability assessment}

The system reliability is discussed in this section. Figure 1 presents the time-dependent failure probabilities $P_{\mathrm{f}, k n}(T)=1-L_{k n}(T)$ for reference periods up to 50 years associated with a single component and a 3 -out-of- 5 system with $k=3$ and a linear deterioration function. The two live load models as summarized in Table 1 are considered. Note that $\mathbf{a}(0)=\mathbf{R}$ since $c_{j}=1$ for each $j$. The probability of failure of a single component is calculated according to Eq. (2), while the system failure probability is estimated with 100,000 replications of simulation. From Fig. 1 it is seen that, for either a component or a system, a greater load intensity leads to a greater probability of failure as expected. Furthermore, for either a component or a system, the logarithm of failure probability increases approximately linearly with the duration of reference period after 20 years. In the presence of the same load condition, the system reliability is greater than that of a single component because of the redundancy of 


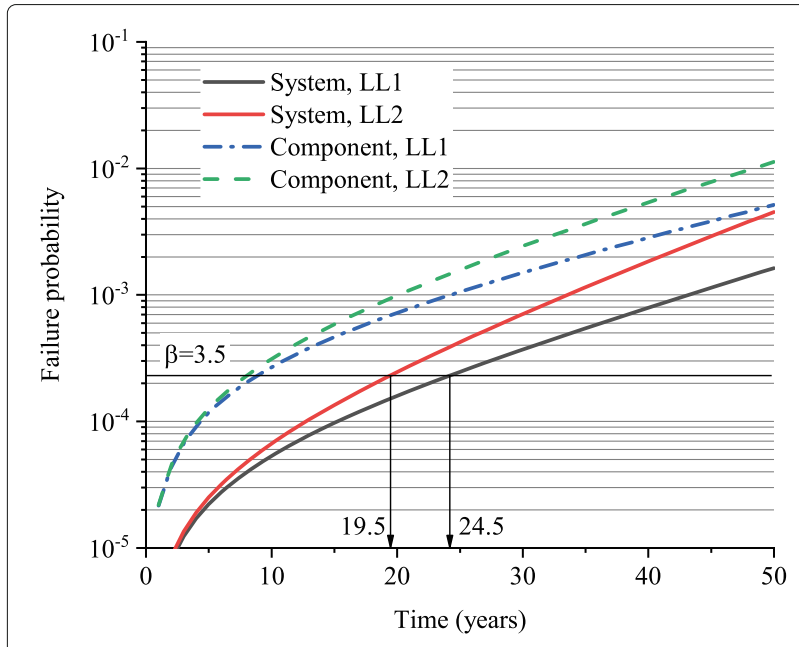

Fig. 1 Time-dependent failure probabilities of a 3-out-of-5 system and a single component the 3-out-of-5 system. In fact, as shown in Table 2, the mean value of $\mathcal{M}(\mathbf{R}, 3)$ is close to that of component resistance, while the standard deviation of $\mathcal{M}(\mathbf{R}, 3)$ is smaller, yielding a greater system reliability.

One can use the results in Fig. 1 to estimate the system's performance (e.g., the service life) under the context of reliability-based assessment. For instance, for the 3out-of- 5 system with a target reliability index of $\beta=3.5$ (corresponding to a failure probability of $2.33 \times 10^{-4}$ ), the service lives associated with live load models 1 and 2 (c.f. Table 1) are 24.5 and 19.5 years respectively. The difference between the predicted service lives is evident of the importance of considering the future varying trend of loads in an attempt to reasonably estimate the system performance.

The time-dependent failure probabilities for 4-out-of5 and 5-out-of-5 (series) systems are presented in Figs. 2 and 3, respectively. The deterioration shape is linear for all components and the two live load models in Table 1 (LL1 and LL2) are used. Similar to Fig. 1, a greater load intensity results in a lower reliability due to the enhanced load risks. The failure probability of a 4-out-of- 5 system is close to that of a single component, due to the closeness between the probabilistic behaviour of $\mathcal{M}(\mathbf{R}, 4)$ and component resistance at the lower tail. As shown in Table 2, both the

Table 2 Mean and standard deviation $(\mathrm{kN} \cdot \mathrm{m})$ of $\mathcal{M}(\mathbf{R}, \mathrm{k})$ and component resistance

\begin{tabular}{lll}
\hline Item & Mean & Standard deviation \\
\hline Component resistance & 3600 & 720 \\
$\mathcal{M}(\mathbf{R}, 3)$ & 3574.7 & 573.3 \\
$\mathcal{M}(\mathbf{R}, 4)$ & 3338.4 & 539.5 \\
$\mathcal{M}(\mathbf{R}, 5)$ & 3045.0 & 515.6 \\
\hline
\end{tabular}

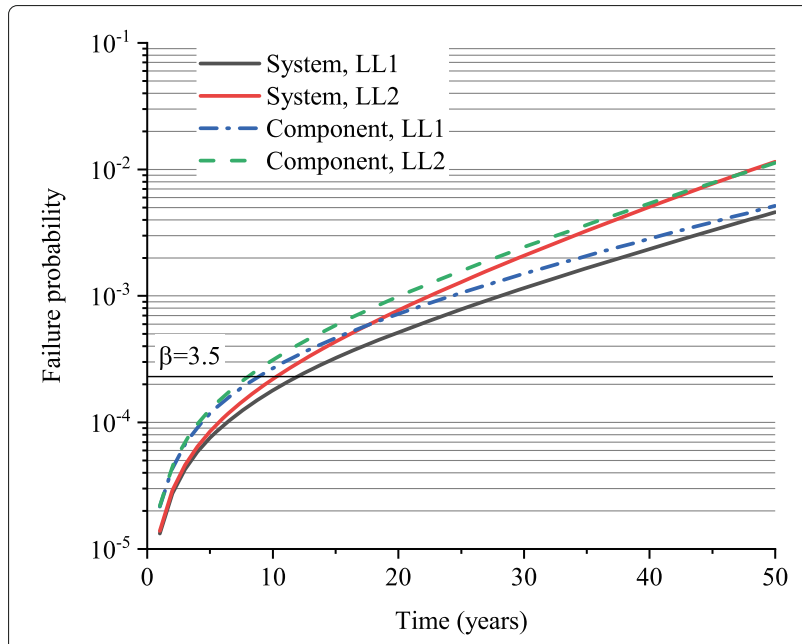

Fig. 2 Time-dependent failure probabilities of a 4-out-of-5 system and a single component

mean value and standard deviation of $\mathcal{M}(\mathbf{R}, 4)$ are smaller than those of the component resistance, and these two factors have opposite effects on the system reliability. In Fig. 3, the probability of failure of a series system is greater than that of a single component, which is consistent with that reported in [20].

Comparing Figs. 1 through 3 it can be seen that when $n$ is fixed, a greater $k$ results in a smaller reliability. Correspondingly, with a target reliability index of 3.5 , the predicted service life of the system is shortened with a greater value of $k$. This can be explained by observing the monotonicity of $\mathcal{M}$. In fact, for two integers $k_{1}$ and $k_{2}$ satisfying $1 \leq k_{1} \leq k_{2} \leq n$, it follows that $\mathcal{M}\left(\mathbf{a}, k_{1}\right) \geq$ $\mathcal{M}\left(\mathbf{a}, k_{2}\right)$ by the definition of $\mathcal{M}$.

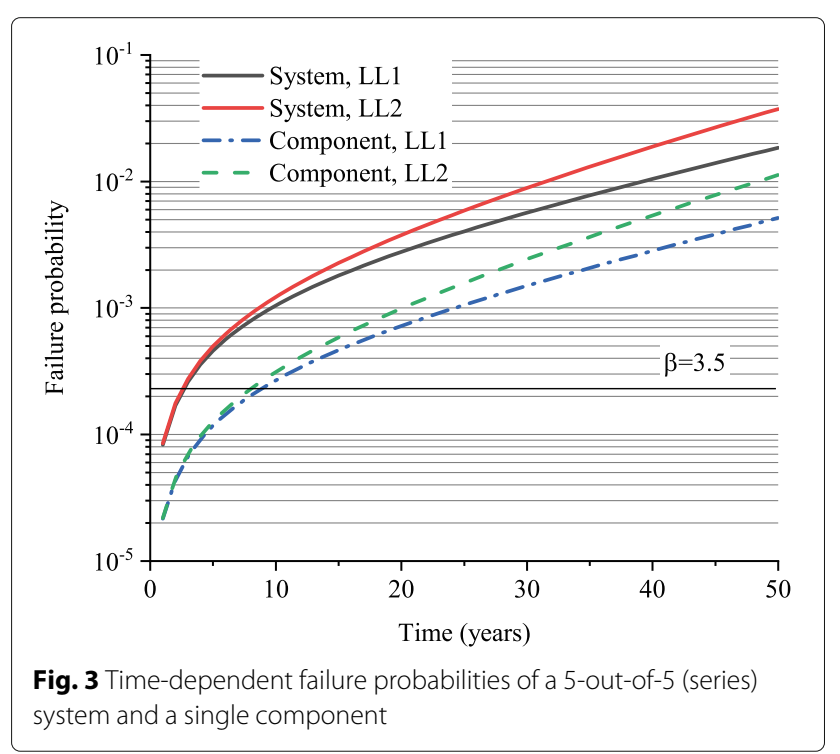




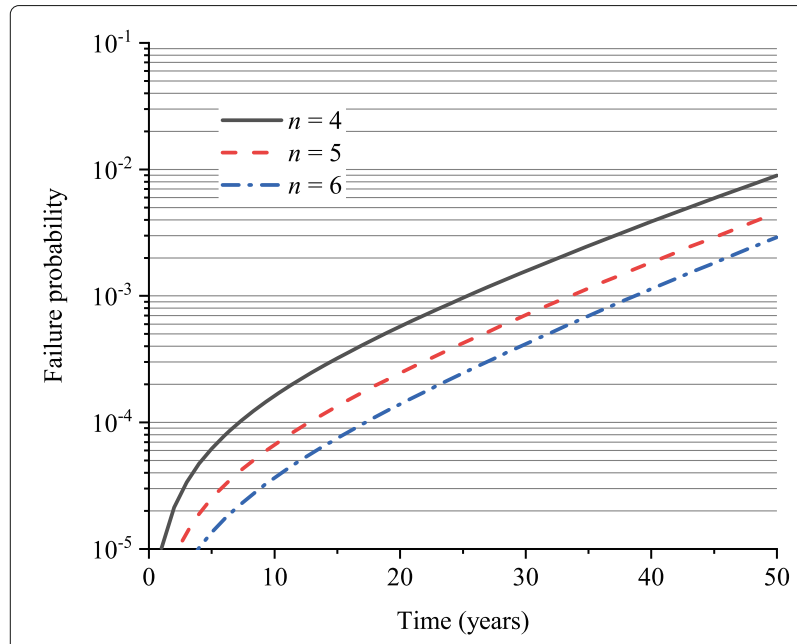

Fig. 4 Time-dependent failure probabilities of 3-out-of-n systems for different numbers of component

The dependence of system failure probability on the number of components, $n$, is presented in Fig. 4 , where the load model LL2 in Table 1 is used. A greater number of components results in a smaller failure probability of the system, due to the enhanced system redundancy.

The time-dependent failure probabilities for a weighted $k$-out-of-5 system are shown in Fig. 5, with a linear deterioration model and LL2. It is assumed that the weights for the five components are 1,2,3,4 and 5 respectively. Note that in this case, $k$ could be greater than $n$ while $k_{s}$ varies between 1 and $n$. It is observed from Fig. 5 that a smaller value of $k$ gives a smaller failure probability due to the weaker requirement of system survival. Furthermore, with

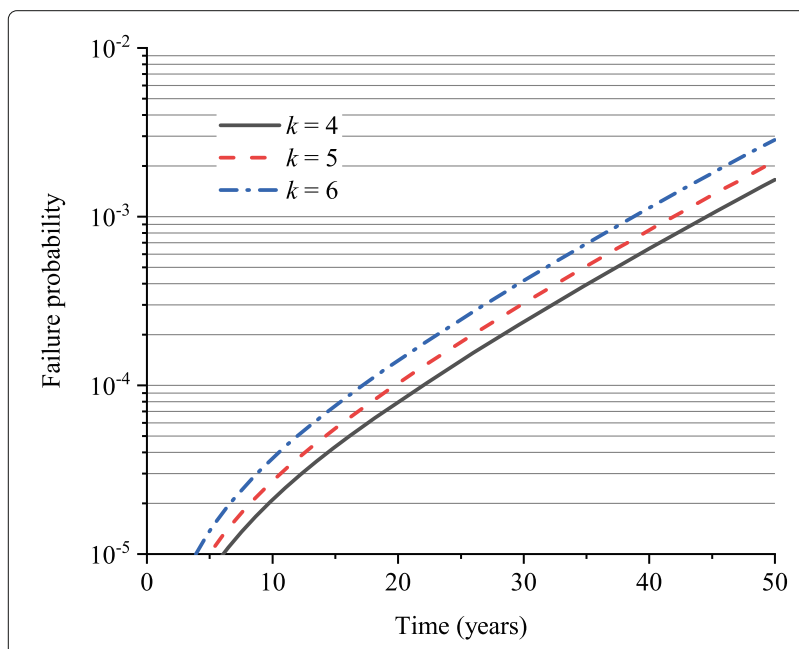

Fig. 5 Time-dependent failure probabilities of weighted $k$-out-of-5 systems for different $k$

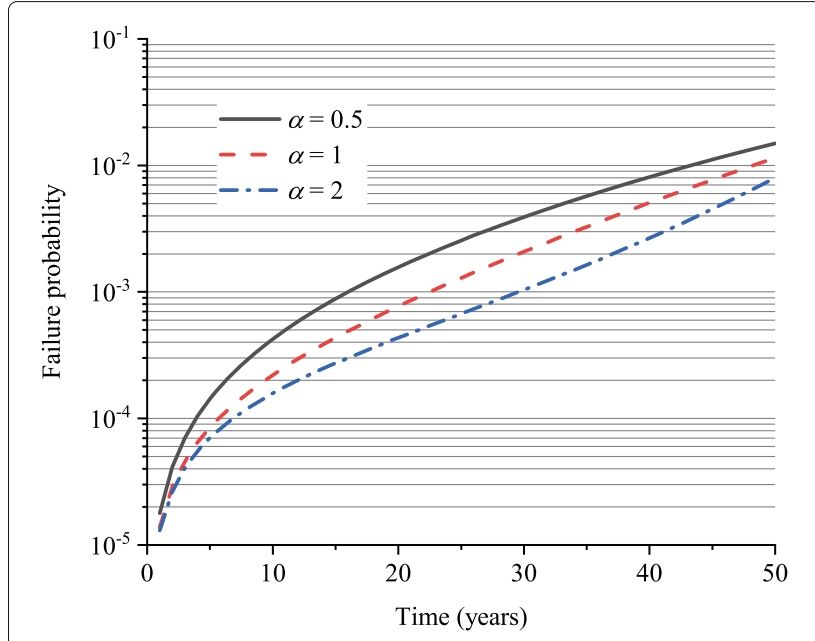

Fig. 6 Dependence of failure probability of a 4-out-of-5 system on deterioration shape with $g(50)=0.8$

both $k$ and $n$ fixed, the system failure probability associated with a weighted system is smaller than that of an ordinary system because $k_{s} \leq k$.

\section{Roles of resistance correlation and deterioration}

Figures 6 and 7 examine the effect of resistance deterioration function on the time-dependent reliability of a 4-out-of-5 system, where LL2 in Table 1 is used. The component resistance degrades by $20 \%$ over 50 years in Fig. 6 and 30\% in Fig. 7. The different values of $\alpha$ indicate different dominant deterioration mechanisms. It can be seen that a severer resistance deterioration leads to a greater failure probability due to the increased probability of load effect exceeding the resistance. Furthermore, a square-root deterioration shape (with $\alpha=0.5$ ) results in the greatest failure probability, followed by linear and parabolic deterioration models. This is because, with the same $g(50)$, the deterioration mainly occurs at the early stage with $\alpha=0.5$, which gives the smallest resistance over the time period of 50 years.

The time-dependent failure probabilities associated with a weighted 4-out-of-5 system subjected to LL2 are presented in Figs. 8 and 9, where the weights for the five components are 1 through 5 respectively. It is observed that a severer resistance deterioration or a smaller value of $\alpha$ leads to a greater failure probability, which is consistent with the observations from Figs. 6 and 7. Furthermore, the failure probability of a weighted system is smaller than that of an ordinary one due to the fact that $k_{s} \leq k$.

Figures 10 and 11 show the dependence of timedependent 4-out-of-5 system failure probability on the resistance correlation $\rho$. The LL2 in Table 1 is used and the component resistance degrades linearly by $20 \%$ over 50 years. The system is an ordinary one in Fig. 10 and 


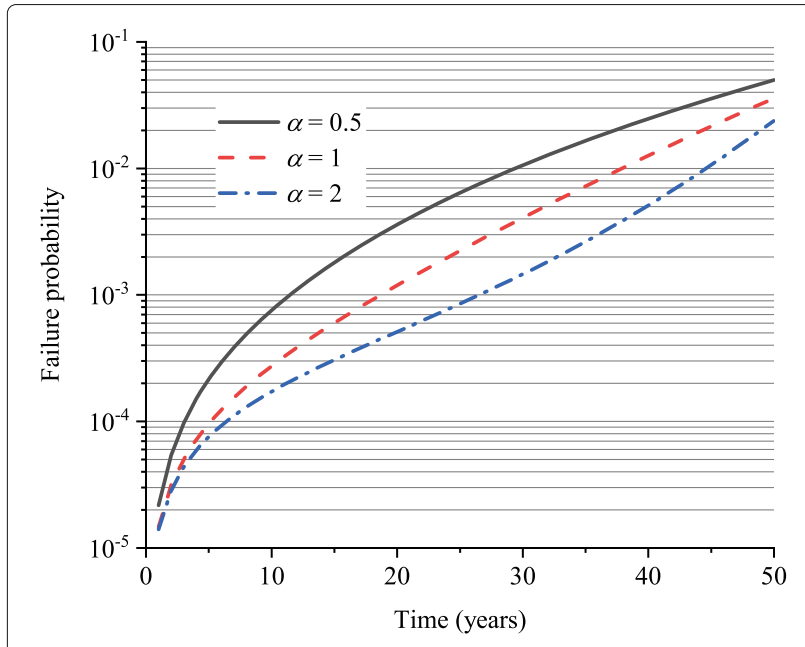

Fig. 7 As of Fig. 6 but with $g(50)=0.7$

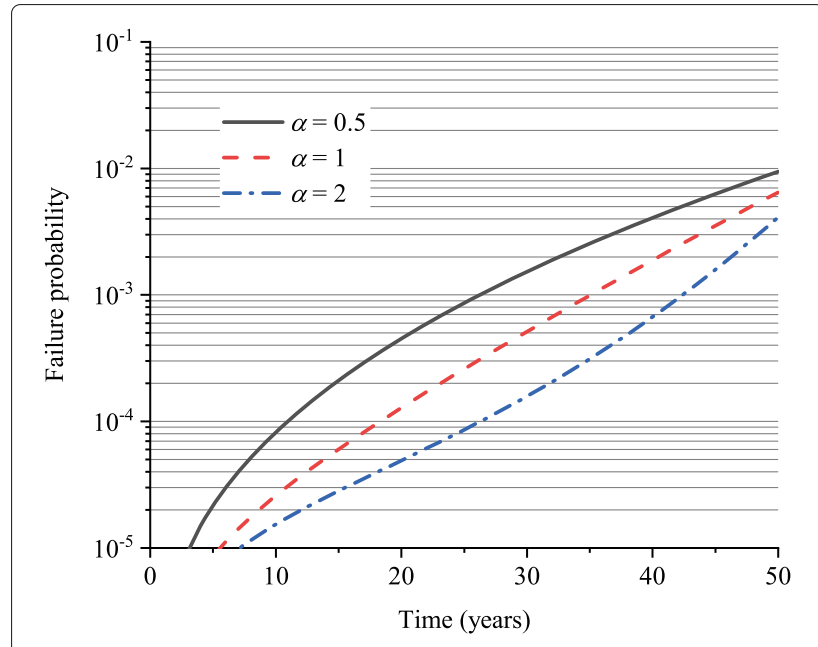

Fig. 9 As of Fig. 8 but with $g(50)=0.7$ weighted in Fig. 11. A greater resistance correlation results in a greater failure probability due to the increased variation of $\mathcal{M}(\mathbf{R}, 4)$. In fact, as $\rho$ increases from 0.3 to 0.9 , the standard deviation of $\mathcal{M}(\mathbf{R}, 4)$ increases from 471.3 to $673.7 \mathrm{kN} \cdot \mathrm{m}$. While the mean of $\mathcal{M}(\mathbf{R}, 4)$ increases from 3286.0 to $3486.0 \mathrm{kN} \cdot \mathrm{m}$ simultaneously, the system reliability is less sensitive to this increase in mean. However, the relationship between the system reliability and the resistance correlation, as revealed in Figs. 10 and 11, does not necessarily hold for any $k$. For example, Fig. 12 shows the time-dependent failure probability for a 5 -out-of- 5 (series) system, where the structural configuration is the same as that in Fig. 10. It can be seen that a greater resistance correlation results in a smaller failure probability due to the fact that the increase of mean value of $\mathcal{M}(\mathbf{R}, 5)$ dominates in the system reliability compared with the increase

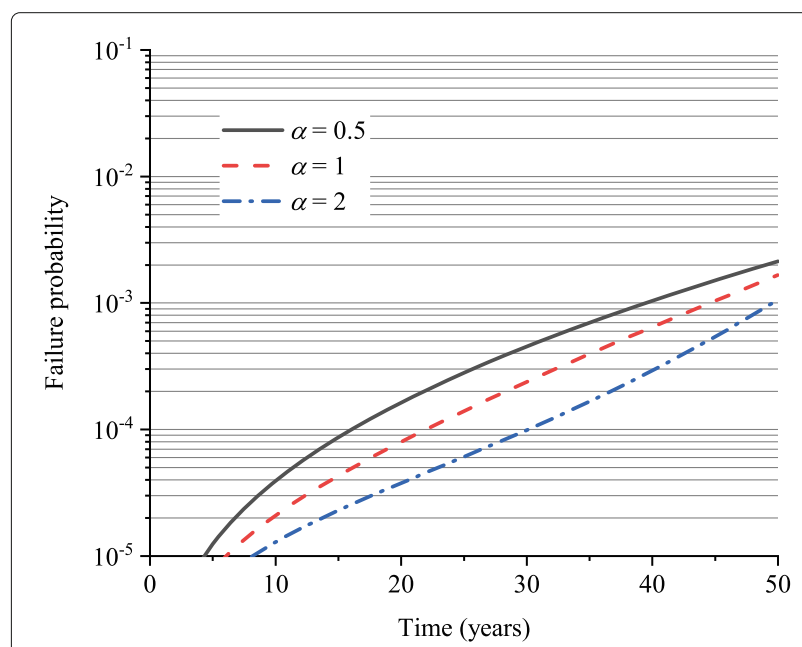

Fig. 8 Dependence of failure probability of a weighted 4-out-of-5 system on deterioration shape with $g(50)=0.8$ of standard deviation. Thus, one should carefully identify the resistance correlation when assessing the system reliability in practice.

Finally, it is noticed that for a $k$-out-of- $n$ system, the reliabilities with $k=1$ (parallel system) and $k=n$ (series system) are the upper and lower bounds for the system reliability, respectively, if the resistance deterioration for each component is identical. This can be seen by recalling Eq. (12), where $\mathcal{M}(\mathbf{a}(0), n) \leq \mathcal{M}(\mathbf{a}(0), k) \leq \mathcal{M}(\mathbf{a}(0), 1)$. A more rigorous proof is as follows. Since

$$
\mathcal{M}(\mathbf{a}(t), n) \leq \mathcal{M}(\mathbf{a}(t), k) \leq \mathcal{M}(\mathbf{a}(t), 1)
$$

it follows that,

$F_{S}[\mathcal{M}(\mathbf{a}(t), n), t] \leq F_{S}[\mathcal{M}(\mathbf{a}(t), k), t] \leq F_{S}[\mathcal{M}(\mathbf{a}(t), 1), t]$,

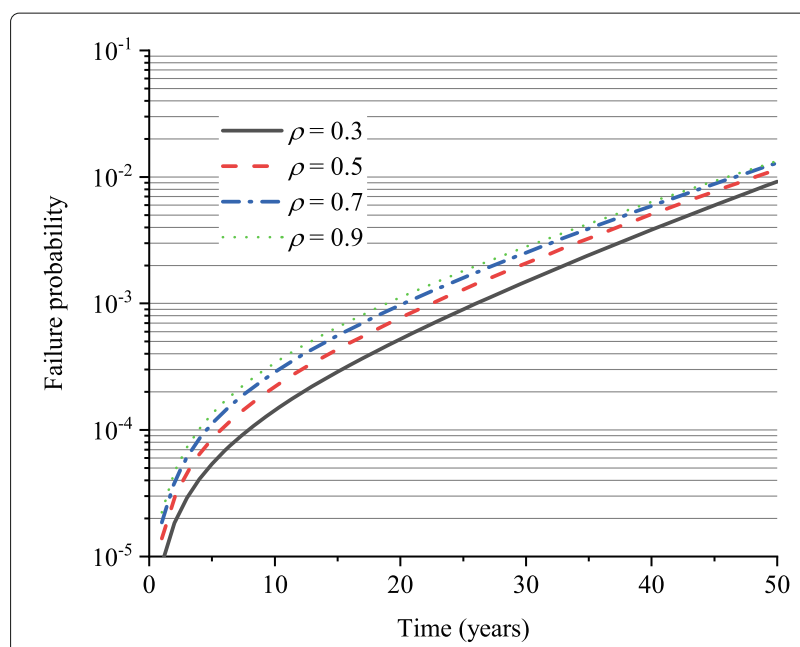

Fig. 10 Dependence of failure probability of a 4-out-of-5 system on component resistance correlation $(\rho)$ 


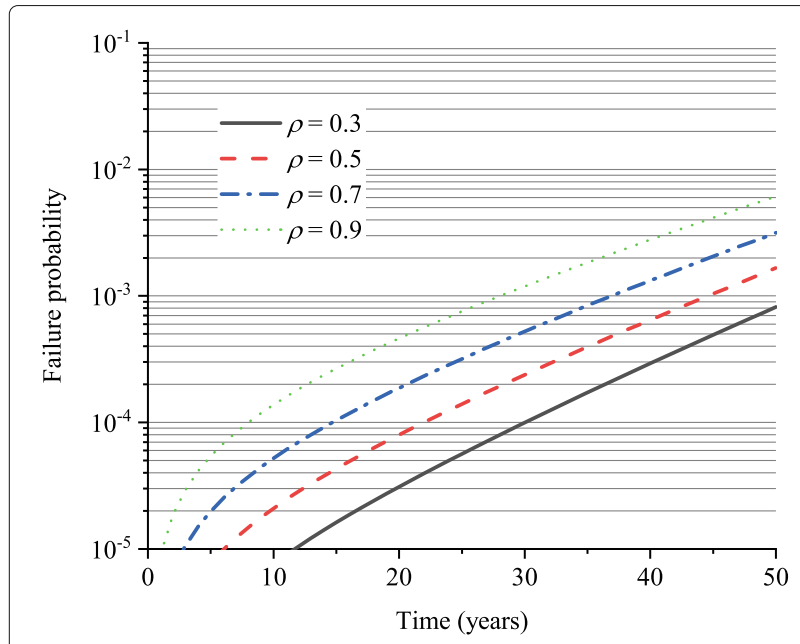

Fig. 11 Dependence of failure probability of a weighted 4-out-of-5 system on $\rho$

or equivalently,

$F_{S}\left[\mathcal{M}(\min (\mathbf{a}(t)), t] \leq F_{S}[\mathcal{M}(\mathbf{a}(t), k), t] \leq F_{S}[\max (\mathbf{a}(t)), t]\right.$.

According to Eq. (7), one has

$$
h_{p s}(t) \leq h_{k n}(t) \leq h_{s s}(t)
$$

with which

$$
L_{s s}(T) \leq L_{k n}(T) \leq L_{p s}(T)
$$

This relationship can be further extended to a weighted $k$-out-of- $n$ system, and the proof is similar (by replacing $k$ with $k_{s}$, which varies between 1 and $n$ at any time $t$ ).

\section{Concluding remarks}

In this paper, a new method is presented for the estimation of time-dependent reliability of aging $k$-out-of- $n$ systems

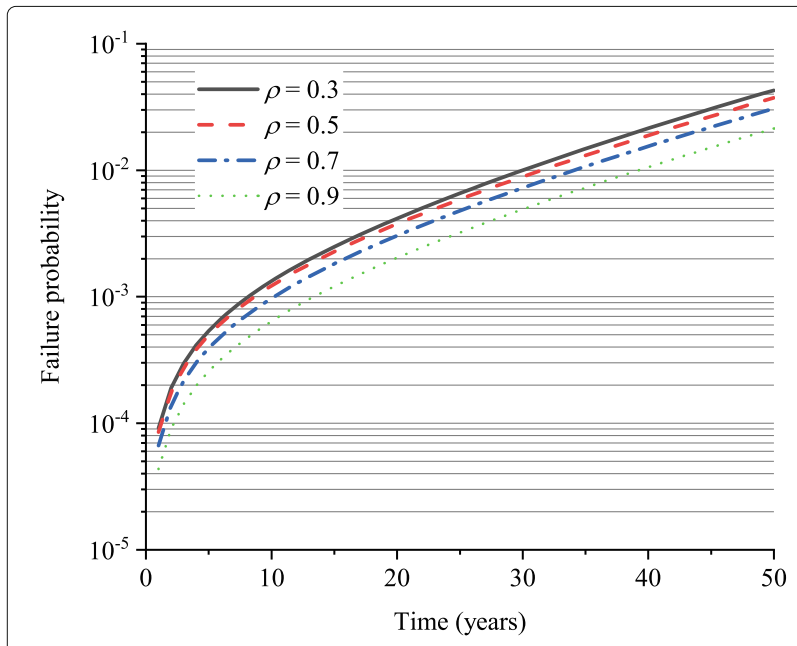

Fig. 12 As of Fig. 10 but with a 5-out-of-5 system (for both ordinary and weighted ones), considering the non-stationarity in the external loads and the component resistance deterioration and correlation. An illustrative example is presented to demonstrate the applicability of the proposed method.

Analytical results show that, for a component or a $k$-outof- $n$ system (either ordinary or weighted), the increase of mean load intensity has a significant impact on the failure probability. For a $k$-out-of- $n$ system, an increase of component number leads to a smaller failure probability. Furthermore, a severer resistance deterioration or a square-root deterioration shape results in a greater failure probability due to the increased risks of load effect exceeding structural resistance. The reliabilities associated with a parallel system and a series system serve as the upper and lower bounds of the $k$-out-of- $n$ system reliability. However, the relationship between the system reliability and the resistance correlation is not necessarily monotonic, suggesting the importance of reasonably identifying component resistance correlation in the estimate of system safety level.

It is finally noticed that the $k$-out-of- $n$ system considered in this paper has been assumed to have identical component resistance deterioration. More research efforts are needed in the future to generalize the case to that with different deterioration processes of component resistance.

\section{Abbreviations}

CDF: cumulative distribution function; PDF: probability density function

\section{Acknowledgements}

The author would like to acknowledge the thoughtful suggestions of two anonymous reviewers, which substantially improved the present paper.

\section{Authors' contributions}

C.W. wrote the paper. The author read and approved the final manuscript.

\section{Funding}

The research described in this paper was supported by the Vice-Chancellor's Postdoctoral Research Fellowship from the University of Wollongong. This support is gratefully acknowledged.

\section{Availability of data and materials}

All data generated or analysed during this study are included in this published article.

\section{Declarations}

Competing interests

The author declares that he has no competing interests.

Received: 2 January 2021 Accepted: 18 February 2021

Published online: 04 March 2021

\section{References}

1. Enright MP, Frangopol DM (1998) Service-life prediction of deteriorating concrete bridges. J Struct Eng 124(3):309-317

2. Ellingwood BR (2005) Risk-informed condition assessment of civil infrastructure: state of practice and research issues. Struct Infrastruct Eng $1(1): 7-18$ 
3. Melchers RE, Beck AT (2018) Structural Reliability Analysis and Prediction, Third Edition. Wiley, Hoboken

4. Wang C, Zhang H (2018) Roles of load temporal correlation and deterioration-load dependency in structural time-dependent reliability. Comput Struct 194:48-59

5. Bhattacharya B, Li D, Chajes M (2008) Bridge rating using in-service data in the presence of strength deterioration and correlation in load processes. Struct Infrastruct Eng 4(3):237-249

6. Zhong J, Gardoni P, Rosowsky D (2010) Stiffness degradation and time to cracking of cover concrete in reinforced concrete structures subject to corrosion. J Eng Mech 136(2):209-219

7. Ma Y, Zhang J, Wang L, Liu Y (2013) Probabilistic prediction with Bayesian updating for strength degradation of RC bridge beams. Struct Saf 44:102-109

8. Wang C, Zhang H, Li Q (2017) Reliability assessment of aging structures subjected to gradual and shock deteriorations. Reliab Eng Syst Saf 161:78-86

9. Knutson TR, McBride JL, Chan J, Emanuel K, Holland G, Landsea C, Held I, Kossin JP, Srivastava A, Sugi M (2010) Tropical cyclones and climate change. Nat Geosci 3(3):157-163

10. IPCC (2014) Climate change 2014: Synthesis report. Technical report, Contribution of Working Groups I, II and III to the Fifth Assessment Report of the Intergovernmental Panel on Climate Change, IPCC, Geneva, Switzerland:151

11. OBrien EJ, Schmidt F, Hajializadeh D, Zhou X-Y, Enright B, Caprani CC, Wilson S, Sheils E (2015) A review of probabilistic methods of assessment of load effects in bridges. Struct Saf 53:44-56

12. Mori Y, Ellingwood BR (1993) Time-dependent system reliability analysis by adaptive importance sampling. Struct Saf 12(1):59-73

13. Li Q, Wang C, Ellingwood BR (2015) Time-dependent reliability of aging structures in the presence of non-stationary loads and degradation. Struct Saf 52:132-141

14. McAllister T (2013) Developing Guidelines and Standards for Disaster Resilience of the Built Environment: A Research Needs Assessment. US Department of Commerce, National Institute of Standards and Technology

15. Latora V, Marchiori M (2004) How the science of complex networks can help developing strategies against terrorism. Chaos, Solitons Fractals 20(1):69-75

16. Kinney R, Crucitti $P$, Albert R, Latora V (2005) Modeling cascading failures in the North American power grid. Eur Phys J B-Condens Matter Complex Syst 46(1):101-107

17. Doguc O, Ramirez-Marquez JE (2009) A generic method for estimating system reliability using Bayesian networks. Reliab Eng Syst Saf 94(2):542-550

18. Vitoontus S (2012) Risk assessment of building inventories exposed to large scale natural hazards. PhD thesis, Georgia Institute of Technology

19. Zhang L, Wang C (2018) Probability-based practice-oriented seismic behaviour assessment of simply supported RC bridges considering the variation and correlation in pier performance. J Traffic Transp Eng (Engl Ed) 5(6):491-502

20. Wang C, Zhang H, Li Q (2017) Time-dependent reliability assessment of aging series systems subjected to non-stationary loads. Struct Infrastruct Eng 13(12):1513-1522

21. Wang C, Zhang H (2020) Assessing the seismic resilience of power grid systems considering the component deterioration and correlation. ASCE-ASME J Risk Uncertainty Eng Syst B Mech Eng 6(2):020903

22. Wu J-S, Chen R-J (1994) An algorithm for computing the reliability of weighted- $\boldsymbol{k}$-out-of- $\boldsymbol{n}$ systems. IEEE Trans Reliab 43(2):327-328

23. Wang C, Feng K, Zhang H, Li Q (2019) Seismic performance assessment of electric power systems subjected to spatially correlated earthquake excitations. Struct Infrastruct Eng 15(3):351-361

24. Samaniego FJ, Shaked M (2008) Systems with weighted components. Stat Probab Lett 78(6):815-823

25. Amari SV, Bergman R (2008) Reliability analysis of $k$-out-of- $n$ load-sharing systems. In: 2008 Annual Reliability and Maintainability Symposium. IEEE. pp 440-445

26. Mo Y, Xing L, Amari SV, Dugan JB (2015) Efficient analysis of multi-state $k$-out-of- $n$ systems. Reliab Eng Syst Saf 133:95-105

27. Sheng Y, Ke H (2020) Reliability evaluation of uncertain $k$-out-of- $n$ systems with multiple states. Reliab Eng Syst Saf 195:106696
28. Wang Z, Xie L, Li B (2007) Time-dependent reliability models of systems with common cause failure. Int J Performability Eng 3(4):419-430

29. Zhang J, Zhao Y, Ma X (2020) Reliability modeling methods for load-sharing $k$-out-of- $n$ system subject to discrete external load. Reliab Eng Syst Saf 193:106603

30. Chen Y, Yang Q (2005) Reliability of two-stage weighted $k$-out-of- $n$ systems with components in common. IEEE Trans Reliab 54(3):431-440

31. Li W, Zuo MJ (2008) Reliability evaluation of multi-state weighted $k$-out-of- $\boldsymbol{n}$ systems. Reliab Eng Syst Saf 93(1):160-167

32. Eryilmaz S, Tutuncu GY (2009) Reliability evaluation of linear consecutive-weighted- $k$-out-of- $n$ : F system. Asia-Pac J Oper Res 26(06):805-816

33. Eryilmaz S (2013) On reliability analysis of a $\boldsymbol{k}$-out-of- $\boldsymbol{n}$ system with components having random weights. Reliab Eng Syst Saf 109:41-44

34. Faghih-Roohi S, Xie M, Ng KM, Yam RC (2014) Dynamic availability assessment and optimal component design of multi-state weighted $\boldsymbol{k}$-out-of- $\boldsymbol{n}$ systems. Reliab Eng Syst Saf 123:57-62

35. Coit DW, Chatwattanasiri N, Wattanapongsakorn N, Konak A (2015) Dynamic $k$-out-of- $n$ system reliability with component partnership. Reliab Eng Syst Saf 138:82-92

36. Franko C, Tütüncü GY, Eryilmaz S (2017) Reliability of weighted $k$-out-of- $n$ : $G$ systems consisting of two types of components and a cold standby component. Commun Stat-Simul Comput 46(5):4067-4081

37. Zhang Y (2020) Reliability analysis of randomly weighted $k$-out-of- $n$ systems with heterogeneous components. Reliab Eng Syst Saf 205:107184

38. Hamdan K, Tavangar M, Asadi M (2021) Optimal preventive maintenance for repairable weighted k-out-of-n systems. Reliab Eng Syst Saf 205:107267

39. Mori Y, Ellingwood BR (1993) Reliability-based service-life assessment of aging concrete structures. J Struct Eng ASCE 119(5):1600-1621

40. Wang C (2021) Structural Reliability and Time-Dependent Reliability. Springer, Cham

41. Nelsen RB (2007) An Introduction to Copulas, Second Edition. Springer, New York

42. Kumar R, Cline DB, Gardoni P (2015) A stochastic framework to model deterioration in engineering systems. Struct Saf 53:36-43

43. Robert C, Casella G (2013) Monte Carlo Statistical Methods. Springer, New York

44. Zio E (2013) The Monte Carlo Simulation Method for System Reliability and Risk Analysis. Springer, Springer-Verlag London

\section{Publisher's Note}

Springer Nature remains neutral with regard to jurisdictional claims in published maps and institutional affiliations.

\section{Submit your manuscript to a SpringerOpen ${ }^{\circ}$ journal and benefit from:}

- Convenient online submission

- Rigorous peer review

- Open access: articles freely available online

- High visibility within the field

- Retaining the copyright to your article

Submit your next manuscript at $>$ springeropen.com 